\title{
Computation of Risk Severity of the Malicious Node using Adaptive Neuro Fuzzy Inference System (ANFIS)
}

\author{
R. Dharmarajan ${ }^{1}$ and V. Thiagarasu ${ }^{2}$ \\ ${ }^{1}$ Research Scholar, Department of Computer Science, Manonmaniam Sundaranar University, Tamil Nadu \\ ${ }^{2}$ Associate Professor, Department of Computer Science, Gobi Arts and Science College, Erode, Tamil Nadu \\ E-Mail: rd.msu2013@gmail.com
}

\begin{abstract}
The Intrusion Detection System (IDS) can be employed broadly for safety network. Intrusion Detection Systems (IDSs) are commonly positioned alongside with other protecting safety mechanisms, such as authentication and access control, as a subsequent line of defence that guards data structures. In this paper, Adaptive Neuro Fuzzy Inference System has utilized to predict the risk severity of the malicious nodes found the previous classification phase.

Keywords: Wireless Network, Fuzzy Logic, Adaptive Neuro Fuzzy Inference System, Membership Function, KDDCUP dataset, Fuzzy Rules
\end{abstract}

\section{INTRODUCTION}

Fuzzy logic is an addition of Boolean logic suggested in 1965 by Lotfi Zadeh [1] fabricated on the measured theory of fuzzy sets. It is a simplification of the classical set theory. By hosting the perception of degree in the authentication of a state, which empowers a condition to be in a public other than true or false, fuzzy logic delivers a very respected tractability for perceptive, such a feature support us in evaluating the inexactness and qualms involved in a system [2].

Fuzzy logic is based on the theory of fuzzy sets, which is an overview of the classical set theory. The classical sets are also known as opposed to vague, clear sets, and by the same token classical logic is also called as Boolean logic or binary. As said that the theory of fuzzy sets is a broad view of the classical set theory funds that the final is a distinct case of fuzzy sets theory.

\section{FUZZY LOGIC SYSTEM}

A Fuzzy Logic System (FLS) [3] can be demarcated as the nonlinear mapping of an input data set to a scalar output data. A Fuzzy Logic System comprises of four main divisions: inference engine, fuzzifier, rules or Fuzzy Knowledge Base and defuzzifier.

The process of fuzzy logic is elucidated by following process in a stage by stage. The following process epitomizes the Fuzzy Logic System.

Step 1: Define the linguistic variables and terms (initialization).

Step 2: Construct the membership functions (initialization)
Step 3: Construct the rule base (initialization)

Step 4: Convert crisp input data to fuzzy values using the membership functions (fuzzification).

Step 5: Evaluate the rules based on the inference obtained.

Step 6: Combine the results of each rule and formulate the results.

Step 7: Convert the output data to non-fuzzy values (defuzzification).

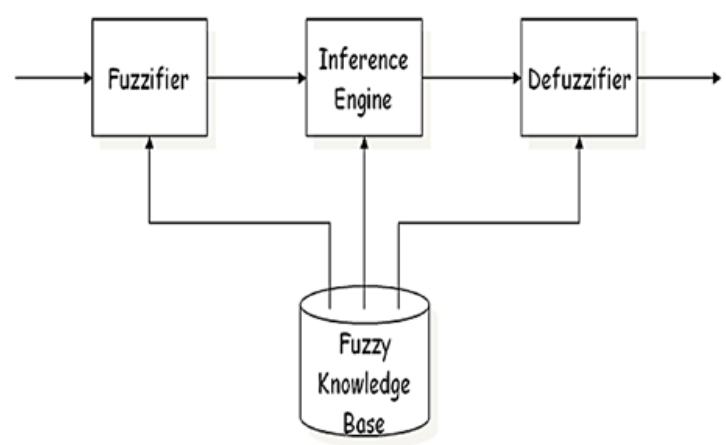

Fig. 1 Components of a Fuzzy Logic System

The steps involved in developing Fuzzy Logic System are as follows:

1. Fuzzifier: Converts the crisp input to a linguistic variable by the membership tasks stored in the fuzzy knowledge base. Fuzzy linguistic variables are accustomed to signify potentials bridging a certain spectrum; e.g. temperature spans are Freezing, Cool, Warm, or Hot.

2. Inference Engine: Using If-Then type fuzzy rules converts the fuzzy input to the fuzzy output.

3. Defuzzifier: Converts the fuzzy output of the inference engine to crisp using membership functions analogous to the ones used by the fuzzifier.

4. Fuzzy Knowledge Base: Information storage for Linguistic variables definitions and Fuzzy rules

\section{A. Linguistic Variable}

Linguistic variables are input or output variables of the Fuzzy system whose values are articulated in a natural language rather than numerical values. A linguistic variable is largely disintegrated into a set of linguistic terms. 


\section{MEMBERSHIP FUNCTION}

A membership function is employed to measure the linguistic term [4]. Membership functions are practiced in the fuzzification and defuzzification steps to record the nonfuzzy input variables to fuzzy linguistic terms and vice versa. There are various methods of membership functions, such as trapezoidal, piecewise linear, triangular, Gaussian or singleton. The optimal of membership functions is context dependent and based on consumer experience. Figure 2 to 5 are portraying the four kinds of membership functions. In the figures, $\mathrm{X}$ axis represents Crisp Values of input and output variables and $\mathrm{Y}$ axis represents membership functions.

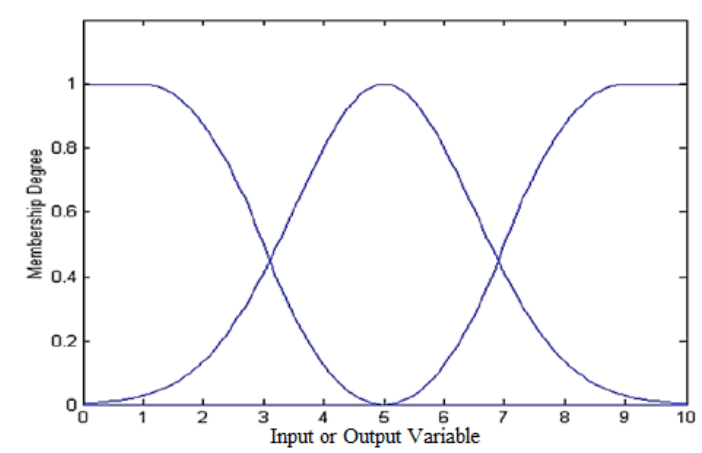

Fig. 2 Gaussian Membership Function X-axis: Crisp Value of Input or Output Variable, Y-axis: Membership Degree

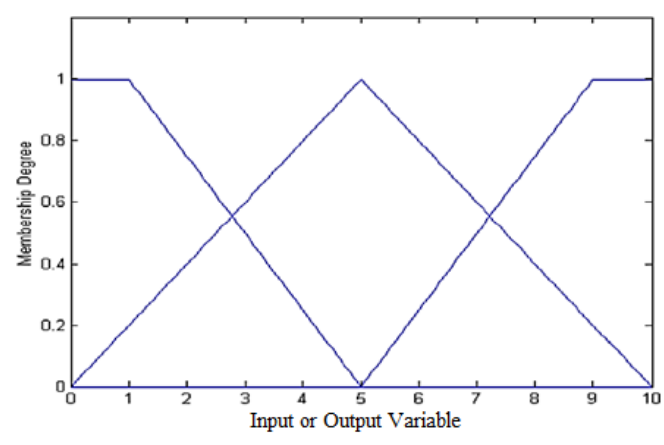

Fig. 3 Triangular Membership Function X-axis: Crisp Value of Input or Output Variable, Y-axis: Membership Degree

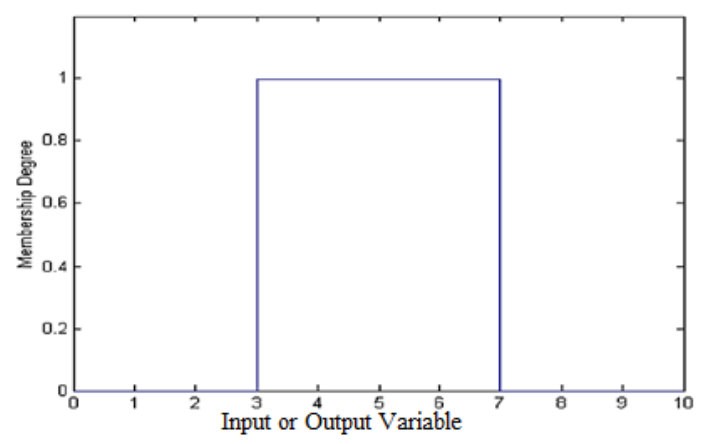

Fig. 4 Rectangular Membership Function X-axis: Crisp Value of Input or Output Variable, Y-axis: Membership Degree

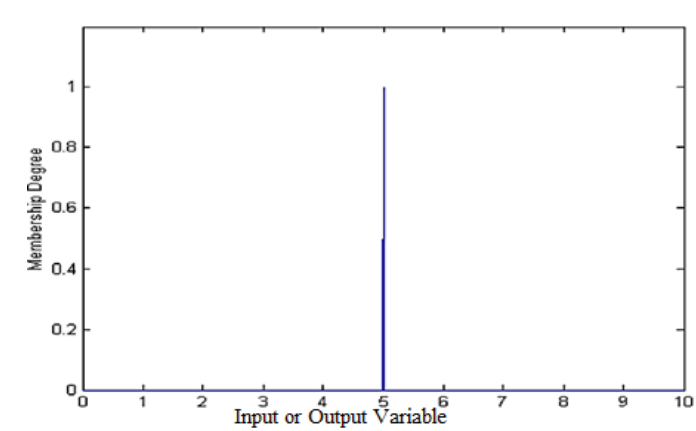

Fig. 5 Singleton Membership Function X-axis: Crisp Value of Input or Output Variable, Y-axis: Membership Degree

\section{FUZZY RULES}

The input to a fuzzy system is named crisp input meanwhile it comprehends accurate information about the constraints. The fuzzifier transforms this exact quantity to an inaccurate quantity like "large", "medium" or "high" with a range of belongingness. Usually, the value ranges from 0 to 1 . A fuzzy rule is a simple IF-THEN rule with a condition and a conclusion. The knowledge base is the major part of a fuzzy system where both data base and rule base are mutually denoted. The data base describes the membership functions of the fuzzy sets practiced in the fuzzy rules, whereas the rule base comprises a number of fuzzy IF-THEN rules.

\section{A. Fuzzy Set Operations}

The assessment of fuzzy rules is accomplished by fuzzy set operations. The frequently cast-off operations for OR and AND operators are min and max, correspondingly. The outcomes of dissimilar rules are pooled to attain a final result after the assessment; this stage known as inference in FLS.

\section{B. Defuzzification}

The fuzzy value is attained by the inference step. This value should be defuzzified to achieve the ultimate crisp output. Defuzzification is accomplished along with the membership function of the output variable. Numerous algorithms are accessible for defuzzification. The best frequently used algorithms are centre of gravity for singletons, centre of gravity, left most maximum and right most maximum.

Two fuzzy inference systems are well known: Sugeno fuzzy model and Mamdani fuzzy model. The Mamdani fuzzy model is created on the assortment of IF-THEN rules together with consequent predicts and fuzzy antecedent. Owing to simplicity of procedure, the Mamdani model is most normally used for answering various real-life complications.

\section{RELATED WORKS}

In the paper [1] debated the complications of neuro-fuzzy modeling and similarly the course for its forthcoming usage. The paper [2] offered a technique where the system was 
observed as a fuzzy model which gave intuition into the real system and correspondingly delivered a technique to streamline the neural network. In the paper [3] projected a modest way for the assortment of inputs for the neuro-fuzzy model in classifying a nonlinear system. The paper [4] recommended additional technique for the selection of inputs of the neuro-fuzzy model fabricated for nonlinear system identification. The papers [5][6] exhibited the solicitation of the neuro-fuzzy method for the demonstrating of nonlinear systems. The paper [7] proposed a technique where the neural network is accustomed by exploiting the numerical data and moreover manual expert knowledge that is epitomized by the fuzzy if and then rules.

In the paper [8] projected a technique for the credentials of a vigorous classification with the assistance of a TakagiSugeno-Kang (TSK) brand fuzzy rule based model which also retains the learning capability of the neural network. In the paper [9] recommended a technique for the identification of a nonlinear system by a fast and stable neuro-fuzzy technique consuming error minimization. In the paper [10] offered a neuro-fuzzy method for the identification of a nonlinear system where in the initial stage the structure identification task is achieved and in the subsequent stage the parameter identification is accomplished. The authors in the [11] also projected a soft calculating based method for the identification of a nonlinear system.

In the paper [12] proposed a method of creating the fuzzy rules by a comprehensive dynamic fuzzy neural network which is erected on the ellipsoidal basis function. The authors in the paper [13] proposed a healthy adaptive fuzzy neural model for the detection of a specific group of multi input-multi output (MIMO) systems. This method has a fast-online learning competence where the fuzzy rules are produced or scrubbed robotically. In the paper [14] proposed a clustering method which is functioned to a combined input output space for the Neuro Fuzzy modeling of nonlinear systems. The experts in paper [15] employed the Neuro-Fuzzy approach for exhibiting the electricity demand in Victoria.

\section{A. Adaptive Neuro Fuzzy Inference System (Anfis) Method for the Risk Severity Prediction of Malicious Nodes}

A Neuro-Fuzzy technique called Adaptive Network based Fuzzy Inference System (ANFIS) has been practiced as a primary device in the current investigation. Adaptive Network based Fuzzy Inference System (ANFIS) is a Neuro Fuzzy technique where the mixture is completed between the fuzzy inference system and the neural network. In ANFIS the constraints can be assessed through the Sugeno and Tsukamoto fuzzy models are epitomized by the ANFIS architecture. Yet again, with negligible limitations the ANFIS model resembles the Radial Basis Function Network (RBFN) functionally. This ANFIS methodology encompasses of a hybrid structure of fuzzy logic and neural network technique. The fuzzy logic proceeds into account the fuzziness and vagueness of the structure that is being molded whereas the neural network stretches it logic of flexibility. By this hybrid method, at first a preliminary fuzzy model accompanied by its input variables are derived with the support of the instructions mined from the input output data of the system that is being modeled. Then, the neural network is cast-off to fine tune the guidelines of the initial fuzzy model to produce the final ANFIS model of the system.

\author{
Input: Optimal Dataset \\ Begin \\ $A=$ selected attribute \\ $\mathrm{S}=$ subset of operation \\ $\mathrm{K}=$ next element from the available data \\ $\mathrm{S}=$ item[i] \\ For $\mathrm{i}=1$ to $\mathrm{n}-1$ \\ $\mathrm{K}=$ DataFIeld $[\mathrm{i}+1]$ \\ $\mathrm{S}=\mathrm{S}$ union $\mathrm{K}$ Select unique item of the
}

field

End for

Store $\mathrm{S}$

End

Initialize Increment to 1

Initialize Weight of Find Record to 0

Initialize Qcnt to 1

WHILE Increment $<$ NI

FOR each value FL END FOR

Index $[\mathrm{FL}]=\operatorname{rand}() \bmod \mathrm{Nfl}$

FOR each value IL

QStr = sql select statement where

each

RandAndOr();

$$
\text { Field }[\mathrm{IL}]=\operatorname{Index}[\mathrm{IL}]+\cdots \cdot+
$$

END FOR
TotFR = ExecuteQuery(Qstr)
IF TotFR is non zero THEN
Wht[Qcnt ] = TotR / TotFR
Add 1 to Qcnt
ENDIF

Add 1 to Increment

ENDWHILE

Save Wht

Save Qstr

Output: Attack Severity: Stage 1 (Low), Stage 2 (Medium) and Stage 3 (High)

\section{RESULTS AND DISCUSSION}

ANFIS is employed with MATLAB R2018b for the risk severity prediction of malicious node detected in the preceding arrangement ANN method.

\section{A. Reduced Dataset}

Following table depicts the reduced dataset acquired by projected pre-processing for Intrusion Detection System Feature selection and Ant Gain Classification Framework [R]. 
TABLE I REDUCED DATASET ObTAINED By PRoposed ANT GAIN FEATURE SELECTION AND ClASSIFICATION FRAMEWORK FOR IDS [R]

\begin{tabular}{|c|c|}
\hline S. No. & Features obtained \\
\hline 1 & Duration \\
\hline 2 & Src_bytes \\
\hline 3 & Dst_bytes \\
\hline 4 & Dst_bytes \\
\hline 5 & rerror_rate \\
\hline 6 & serror_rate \\
\hline 7 & Service \\
\hline
\end{tabular}

\section{B. Input Membership Function}

1. Source_Bytes: This input variable Source_Bytes signifies the amount of data bytes from source to destination. The succeeding assortments are used to define the source bytes size throughout the broadcast in the network. Table II provides the fuzzy table for input variable source bytes and its membership has denoted in the figure 6.

TABLE II FuZZY TABLE For INPUT VARIABLE SOURCE_ByTES

\begin{tabular}{|c|c|c|}
\hline Input Field & Range & $\begin{array}{c}\text { Linguistic } \\
\text { Representation }\end{array}$ \\
\hline \multirow{3}{*}{ Source_Bytes } & $0-15000$ bytes & Range 1 \\
& $15000-28000$ bytes & Range 2 \\
& $28000-100000$ bytes & Range 3 \\
\hline
\end{tabular}

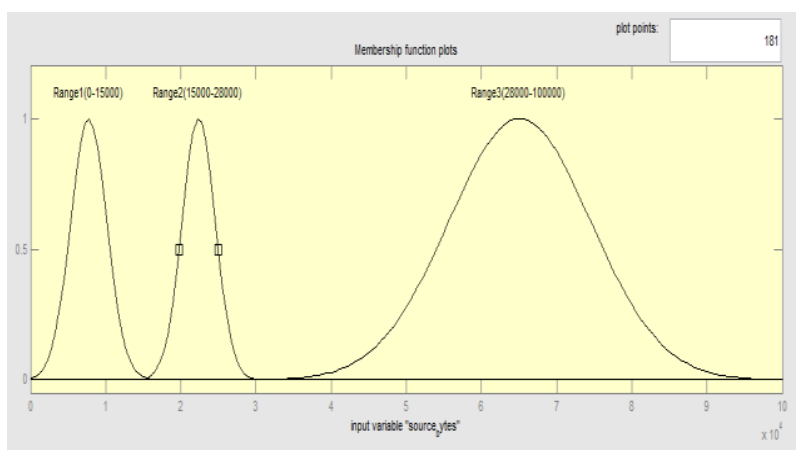

Fig. 6 Membership Function Plot for Input Variable "Source bytes". Xaxis: Input Variable "Source_bytes", Y-axis: Membership Degree of "Source_bytes

2. Destination_Bytes: This input variable Destination_Bytes is cast-off to characterize the amount of data bytes from destination to source. The following ranges are used to pronounce the destination bytes size for the period of transmission in the network. Table III gives the fuzzy table for the input variable destination bytes and its representation of membership has depicted in the figure 7.

TABLE II FUZZY TABLE For INPUT VARIABLE DESTINATION_ByteS

\begin{tabular}{|c|c|c|}
\hline Input Field & Range & $\begin{array}{c}\text { Linguistic } \\
\text { Representation }\end{array}$ \\
\hline \multirow{2}{*}{ Destination_Bytes } & $0-15000$ bytes & Range 1 \\
& $15000-28000$ bytes & Range 2 \\
& $28000-100000$ bytes & Range 3 \\
\hline
\end{tabular}

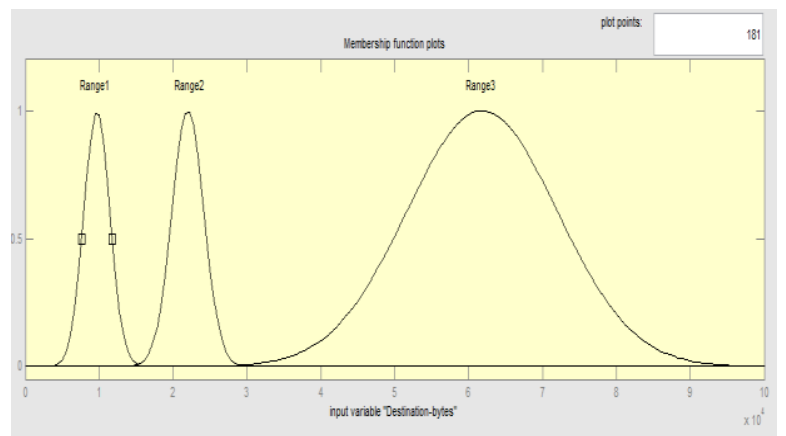

Fig. 7 Membership Function Plot for Input Variable "Destination bytes". X-axis: Input Variable "Destination_bytes", Y-axis: Membership Degree of "Destination_bytes"

3. Protocol_type: This variable Protocol_type represents the type of the protocol, e.g. tcp, udp,icmp etc that to be used in the network. Table IV gives the fuzzy table for the input variable protocol_type and its membership has presented in the figure 8

TABLE IV FUZZY TABLE FOR INPUT VARIABLE PROTOCOL_TYPE

\begin{tabular}{|c|c|c|}
\hline Input Field & Range & Linguistic Representation \\
\hline \multirow{3}{*}{ Protocol_type } & $0-3$ & TCP \\
& $3-6$ & UDP \\
& $5-9.5$ & ICMP \\
\hline
\end{tabular}

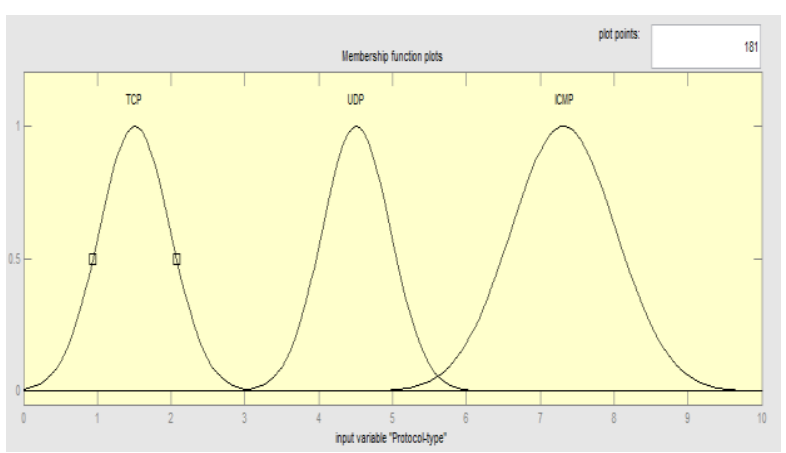

Fig. 8 Membership Function Plot for Input Variable "Protocol_type". Xaxis: Input Variable "Protocol_type", Y-axis: Membership Degree of "Protocol_type"

4. Service: This variable Service represents the network service on the destination, e.g., http, telnet, etc. Table V depicts the fuzzy table for input variable service and figure 9 gives its representation of the membership function.

TABLE V FuZzy TABLE For INPUT VARIABLE SERVICE

\begin{tabular}{|c|c|c|}
\hline Input Field & Range & Linguistic Representation \\
\hline \multirow{3}{*}{ Service } & $0-2$ & HTTP \\
& $2-4$ & FTP \\
& $4-8$ & Telnet \\
\hline
\end{tabular}

5. Duration: This variable Duration represents the length (number of seconds) of the connection in the network. Table VI gives the fuzzy table for input variable duration and its membership function has given in the figure 10 . 


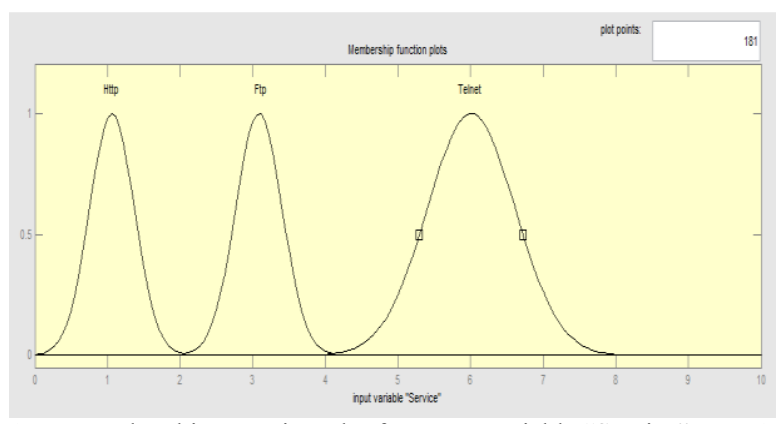

Fig. 9 Membership Function Plot for Input Variable "Service". X-axis: Input Variable "Service", Y-axis: Membership Degree of "Service"

TABLE Vi FUZZY TABLE For INPUT VARIABLE DURATION

\begin{tabular}{|c|c|c|}
\hline Input Field & Range & Linguistic Representation \\
\hline \multirow{3}{*}{ Duration } & $0-10$ Seconds & Time 1 \\
& $11-30$ Seconds & Time 2 \\
& $>30$ Seconds & Time 3 \\
\hline
\end{tabular}

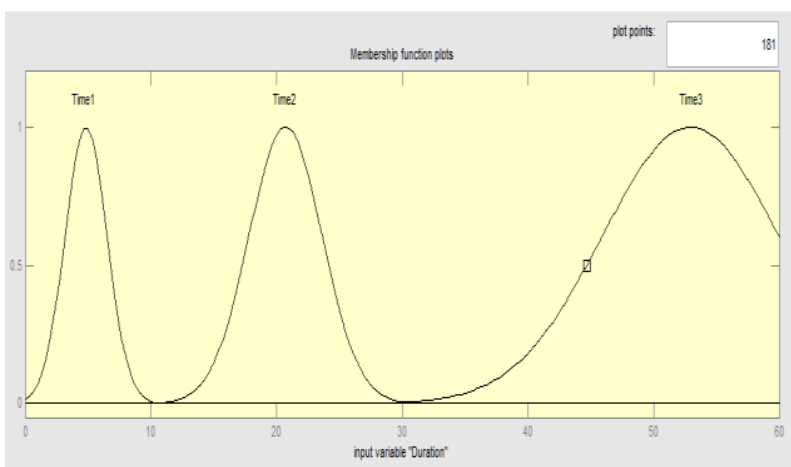

Fig. 10: Membership Function Plot for Input Variable "Duration". X-axis: Input Variable "Duration", Y-axis: Membership Degree of "Duration"

6. Synchronization Error Rate (serror_rate): This input variable Synchronization Error Rate exposes that the \% of connections that have "SYN" Errors in the network. Table VII depicts the fuzzy table for the input variable serror rate and its membership function has portrayed in the figure 11.

TABLE ViI FuZZy TABLE For INPUT VARIABLE SERROR_RATE

\begin{tabular}{|c|c|c|}
\hline Input Field & Range & Linguistic Representation \\
\hline \multirow{3}{*}{ serror_rate } & $0-10 \%$ & Type 1 \\
& $11-30 \%$ & Type 2 \\
& $31-100 \%$ & Type 3 \\
\hline
\end{tabular}

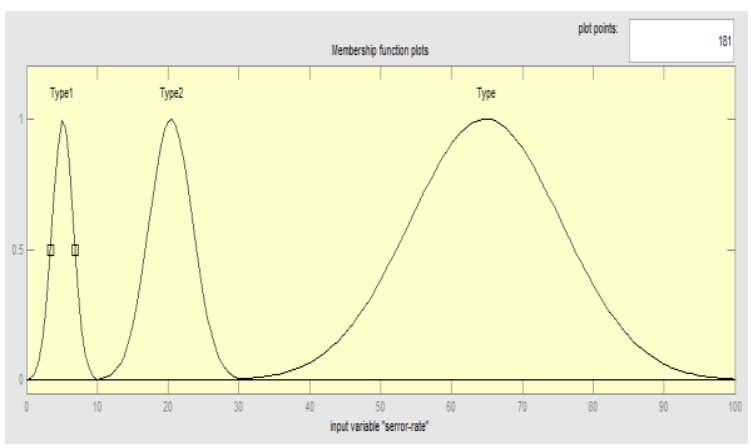

Fig. 11 Membership Function Plot for Input Variable "serror_rate". X-axis: Input Variable "serror_rate", Y-axis: Membership Degree of "serror_rate"
7. rerror_rate (Response Error rate): This input variable Response Error Rate represents that the \% of connections that have "Response" Errors in the network. Table VIII represents the fuzzy table for input variable error_rate and figure 12 gives the membership function for the given input variable.

TABLE VIII FUZZY TABLE For INPUT VARIABLE RERROR_RATE

\begin{tabular}{|c|c|c|}
\hline Input Field & Range & Linguistic Representation \\
\hline \multirow{3}{*}{ rerror_rate } & $0-25 \%$ & Type 1 \\
& $25-50 \%$ & Type 2 \\
& $50-100 \%$ & Type 3 \\
\hline
\end{tabular}

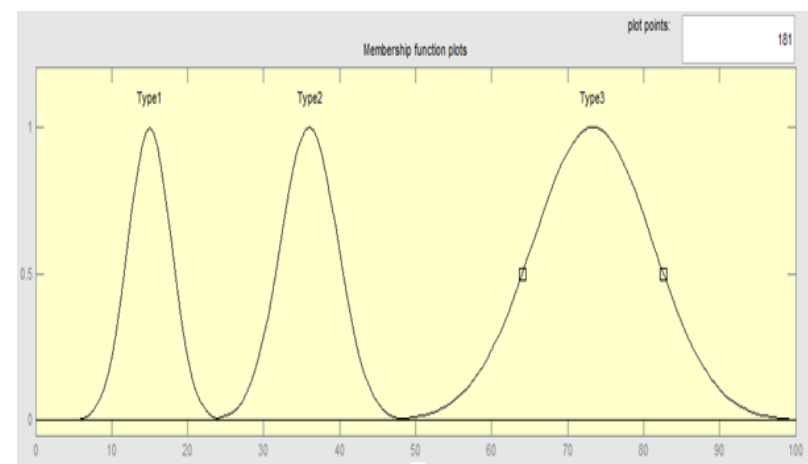

Fig. 12 Membership Function Plot for Input Variable "rerror_rate". X-axis: Input Variable "rerror_rate", Y-axis: Membership Degree of "rerror_rate"

\section{Output Membership Function}

This output variable "Severity" is used to remark the risk severity of the wary node in the network. This severity can be categorized into three phases, Low, Mild and High. These phases portray the severity suspicious of the node. Table IC contributes the fuzzy table for the output variable severity and its membership function has described in the figure 13. Figure 14 gives the rule editor view of the ANFIS employed for the risk severity prediction of the malicious nodes.

TABLE IX FuZZY TABLE FOR OUTPUT VARIABLE SEVERITY

\begin{tabular}{|c|c|c|}
\hline Input Field & Range & Linguistic Representation \\
\hline \multirow{3}{*}{ Severity } & $0-25 \%$ & LOW \\
& $26-40 \%$ & MILD \\
& $41-100 \%$ & TYPE 3 \\
\hline
\end{tabular}

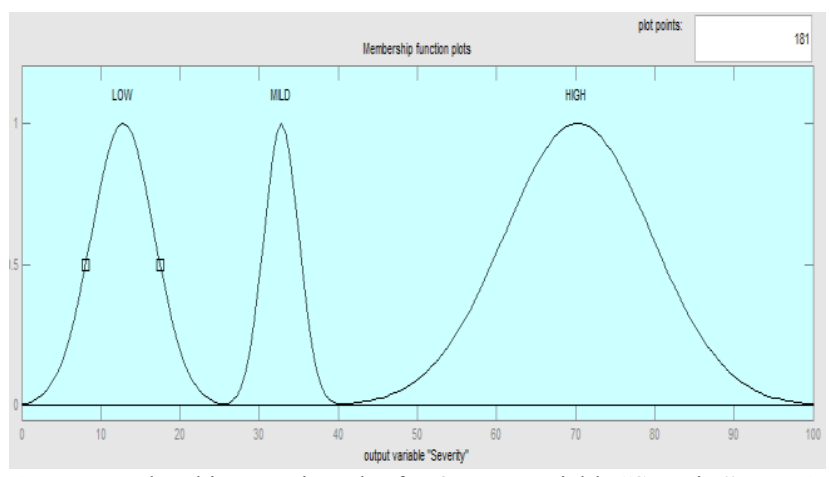

Fig. 13 Membership Function Plot for Output Variable "Severity". X-axis: Input Variable "Severity", Y-axis: Membership Degree of "Severity" 


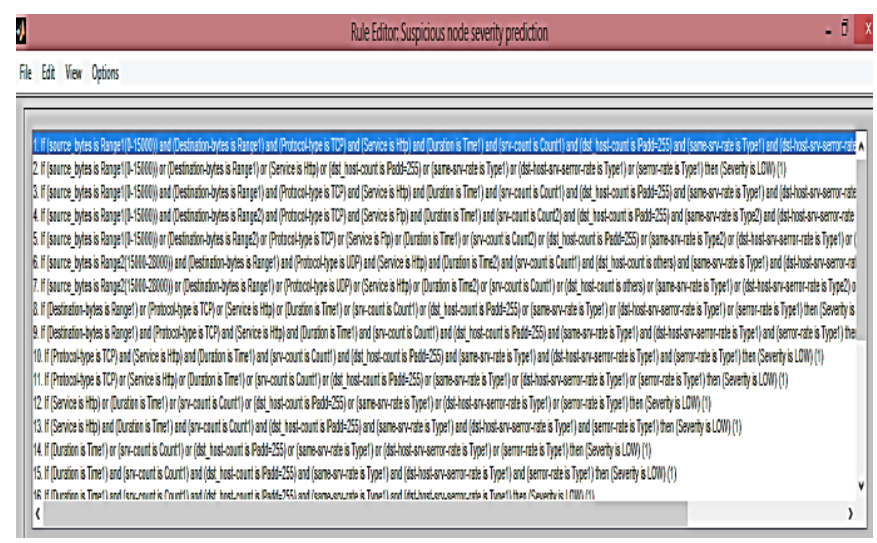

Fig. 14 Rule Editor View of ANFIS in Severity Prediction of Suspicious Node

\section{CONCLUSION}

The major objective of the present research is to detect a suspicious node severity from several factors of KDDCUP 99 dataset which comprises the attacks data, as it may lead a node to malicious or not. The results acquired to designate that the proposed method can be cast-off to induce fuzzy rules from data by delivering respectable balance between precision and readability. Primary prevention is suggested for endorsing good node for direction-finding in network through augmented awareness and consciousness, to inhibit development of any risk factors and a system to assess the prospect of cut in the network for prevention. Based on the risk severity of the malicious node, it will ponder for rerouting. It will augment the efficiency, data processing speed and quality of service of the network.

\section{REFERENCES}

[1] Jang, J-SR, and Chuen-Tsai Sun, "Neuro-fuzzy modeling and control", Proceedings of the IEEE, Vol. 83, No. 3, pp. 378-406, 1995.

[2] Lin, Yinghua, and George A. Cunningham, "A new approach to fuzzy-neural system modeling", IEEE Transactions on Fuzzy systems, Vol.3, No. 2, pp. 190-198, 1995.
[3] Kim, Jaesoo, and Nikola Kasabov, "HyFIS: adaptive neuro-fuzzy inference systems and their application to nonlinear dynamical systems", Neural Networks, Vol. 12, No. 9, pp. 1301-1319, 1999.

[4] Nelles, Oliver, "Nonlinear system identification: from classical approaches to neural networks and fuzzy models", Springer Science \& Business Media, 2013.

[5] C. Nayak, Puma et al., "A neuro-fuzzy computing technique for modeling hydrological time series", Journal of Hydrology, Vol. 291, No. 1-2, pp. 52-66, 2004.

[6] Babuška, Robert, and Henk Verbruggen, "Neuro-fuzzy methods for nonlinear system identification", Annual reviews in control, Vol. 27, No. 1, pp. 73-85, 2003.

[7] Kuo, J. Ren and K. C. Xue, "A decision support system for sales forecasting through fuzzy neural networks with asymmetric fuzzy weights", Decision Support Systems, Vol. 24, No. 2, pp. 105-126, 1998.

[8] Wu, Shiqian, Meng Joo Er, and Yang Gao, "A fast approach for automatic generation of fuzzy rules by generalized dynamic fuzzy neural networks", IEEE Transactions on Fuzzy Systems, Vol. 9, No. 4, pp. 578-594, 2001.

[9] Gao, Yang, and Meng Joo Er, "Online adaptive fuzzy neural identification and control of a class of MIMO nonlinear systems", IEEE Transactions on Fuzzy Systems, Vol. 11, No. 4, pp. 462-477, 2003.

[10] Kukolj, Dragan, and Emil Levi, "Identification of complex systems based on neural and Takagi-Sugeno fuzzy model", IEEE Transactions on Systems, Man, and Cybernetics, Part B (Cybernetics), Vol. 34, No. 1, pp. 272-282, 2004.

[11] Buragohain, Mrinal, and Chitralekha Mahanta, "A novel approach for ANFIS modelling based on full factorial design", Applied soft computing, Vol. 8, No. 1, pp. 609-625, 2008.

[12] Kasabov, K. Nikola and Qun Song, "DENFIS: dynamic evolving neural-fuzzy inference system and its application for time-series prediction", IEEE transactions on Fuzzy Systems, Vol. 10, No. 2, pp. 144-154, 2002.

[13] Bechlioulis, P. Charalampos and George A. Rovithakis, "Robust adaptive control of feedback linearizable MIMO nonlinear systems with prescribed performance", IEEE Transactions on Automatic Control, Vol. 53, No. 9, pp. 2090-2099, 2008.

[14] Abonyi, Janos, Robert Babuska, and Ferenc Szeifert, "Modified Gath-Geva fuzzy clustering for identification of Takagi-Sugeno fuzzy models", IEEE Transactions on Systems, Man, and Cybernetics, Part B (Cybernetics), Vol. 32, No. 5, pp. 612-621, 2002.

[15] Abraham, Ajith, and Baikunth Nath, "A neuro-fuzzy approach for modelling electricity demand in Victoria", Applied Soft Computing, Vol. 1, No. 2, pp. 127-138, 2001. 